\title{
Prolonged low-dose cyclophosphamide treatment after pulse therapy attenuates lung injury in rats with paraquat intoxication
}

\author{
Jie Gao ${ }^{*}$, Shunyi Feng ${ }^{*}$, and Yong Li
}

Department of Emergency, Cangzhou Central Hospital, Cangzhou, China

\author{
Received: October 2, 2017 \\ Revised : December 25, 2017 \\ Accepted: February 2, 2018

\section{Correspondence to} \\ Yong Li, M.D. \\ Department of Emergency, Cang- \\ zhou Central Hospital, No. 16 \\ Xinhua Rd, Yunhe Dist, Cangzhou \\ o61000, China \\ Tel: +86-133-3336-7871 \\ Fax: +86-317-207-5685 \\ E-mail:ly13333367871@hotmail.com
}

*These authors contributed equally to this work.
Background/Aims: This study tested the hypothesis that prolonged low-dose cyclophosphamide (CTX) treatment after pulse therapy attenuate paraquat (PQ)-induced lung injury in rats.

Methods: PQ $(25 \mathrm{mg} / \mathrm{kg})$ was administered intraperitoneally to induce PQ-intoxicated rat model. The rats were randomly divided into four groups: control group (1 mL/day saline solution for 14 days), PQ group (1 mL/day saline solution for 14 days after PQ exposure), pulse group (15 mg/kg/day CTX in $1 \mathrm{~mL}$ of saline solution for 2 days and subsequent $1 \mathrm{~mL} /$ day saline solution for 12 days), and prolonged low-dose group $(15 \mathrm{mg} / \mathrm{kg} /$ day CTX in $1 \mathrm{~mL}$ of saline solution for 2 days and subsequent $1.5 \mathrm{mg} / \mathrm{kg} /$ day CTX in $1 \mathrm{~mL}$ of saline solution for 12 days). A 14day follow-up was conducted to determine the survival rat, and lung hydroxyproline (HYP), wet-to-dry weight ratios (W/Dc) and histopathological changes were evaluated.

Results: Results showed similar survival rate ( $55 \%$ vs. $50 \%$, $p>0.05$ ) between prolonged low-dose and pulse groups. Lung W/Dc ( $4.94 \pm 0.38$ vs. $5.47 \pm 0.28, p<0.01)$, HYP $(3.34 \pm 0.29 \mu \mathrm{g} / \mathrm{mg}$ vs. $3.65 \pm 0.19 \mu \mathrm{g} / \mathrm{mg}, p<0.001)$, and fibrosis score $(2.69 \pm$ 0.84 vs. $3.13 \pm 0.63, p<0.05)$ were lower in prolonged low-dose group than those in the pulse group.

Conclusions: These findings suggested prolonged low-dose CTX treatment after pulse therapy could attenuate $\mathrm{PQ}$-induced lung injury in rats.

Keywords: Paraquat; Cyclophosphamide; Survival rate; Lung fibrosis

\section{INTRODUCTION}

Paraquat (PQ; 1,10-dimethyl-4,40-bipyridinium dichloride) is a highly toxic compound, and its use is rigorously restricted in many industrialized countries, such as the United States, South Korea, and the members of the European Union. Nevertheless, PQ is still widely used as an herbicide in many developing countries worldwide. Several cases of acute poisoning and even death due to this compound have been reported over the past few decades
$[1,2]$. The lung is a main target organ because PQ is actively taken up by the alveolar epithelium, which causes pulmonary injury by acute intoxication or long-term accumulation $[3,4]$. The mechanism of PQ toxicity involves a series of cyclic reduction-oxidation reactions inducing many inflammatory agents, which eventually become progressive lung fibrosis; this mechanism plays an essential role in producing lethal hypoxemia after PQ poisoning $[5,6]$. Cyclophosphamide (CTX) is an alkylating agent with cytotoxic and immunosuppressive properties. CTX, ei- 
ther as a sole agent or in combination with glucocorticoids, is frequently used to treat a variety of inflammatory and fibrosis conditions $[7,8]$. Although prolonged low-dose CTX provides potential benefits in previous case and nonrandomized trials with a small sample size [9-12], the possibility of spontaneous recovery is undetermined. Given the lack of strong evidence of prolonged low-dose CTX therapy for PQ-induced lung injury, the beneficial effects are still unknown.

In the present study, we determined whether the prolonged low-dose CTX treatment after pulse could attenuate PQ-induced lung injury in rats. This study also aimed to observe its potential protective effects through survival rate, lung wet-to-dry weight (W/Dc), hydroxyproline (HYP) content, and fibrosis score.

\section{METHODS}

\section{Chemicals}

HYP was purchased from Sigma-Aldrich Chemical Company (St. Louis, MO, USA). PQ was obtained from Syngenta Chemical(Jiangsu, China). CTX was purchased from Suncadia Pharma (Jiangsu, China).

\section{Animals}

Eighty healthy Wistar rats (200 to $250 \mathrm{~g}$ ) from the Experimental Animal Center were acclimatized to our laboratory environment for 7 days. All rats were kept under environmentally controlled conditions in an air-conditioned room at $23^{\circ} \mathrm{C} \pm 2{ }^{\circ} \mathrm{C}$, with appropriate humidity and a 12-hour:12-hour light:dark cycle. These rats were fed with standard rat food and water ad libitum. This study was carried out in strict accordance with the recommendations in the Guide for the Care and Use of Laboratory Animals of the National Institute of Health. All experimental protocols were approved by the Ethics Committee of Cangzhou Central Hospital, China (Permit Number: 2016-110-02). All surgery was performed under sodium pentobarbital anesthesia, and all efforts were made to minimize suffering.

\section{Experimental design}

PQ poisoning model was established by intraperitoneal injection with $1 \mathrm{~mL}$ of $25 \mathrm{mg} / \mathrm{kg}$ [13,14] body weight of PQ solution; after 60 minutes, the drug or saline solu- tion was administered to the animals through intraperitoneal injection. Based on the results from the existing literature regarding the administration to $\mathrm{PQ}$-intoxicated rats, we defined pulse treatment as $15 \mathrm{mg} / \mathrm{kg} /$ day CTX for 2 days $[15,16]$ and prolonged low-dose therapy as $1.5 \mathrm{mg} / \mathrm{kg} /$ day CTX for 12 days $[16,17]$. The rats were randomly divided into four groups, and each group contained 20 rats. In the control group, rats were intraperitoneally injected with $1 \mathrm{~mL} /$ day saline solution for 14 days. In the PQ group, rats were intraperitoneally injected with $1 \mathrm{~mL}$ /day saline solution for 14 days after PQ exposure. In CTX pulse group, PQ-intoxicated rats were intraperitoneally injected with $15 \mathrm{mg} / \mathrm{kg} /$ day CTX in 1 $\mathrm{mL}$ of saline solution for 2 days and subsequent $1 \mathrm{~mL} /$ day saline solution for 12 days. In prolonged low-dose group, PQ-intoxicated rats were intraperitoneally injected with $15 \mathrm{mg} / \mathrm{kg} /$ day CTX in $1 \mathrm{~mL}$ of saline solution for 2 days and subsequent $1.5 \mathrm{mg} / \mathrm{kg} /$ day CTX in $1 \mathrm{~mL}$ of saline solution for 12 days. All animals were sacrificed 15 days after PQ exposure.

\section{Sample collection}

Prior to the sacrifice by cervical dislocation, rats were anesthetized with phenobarbital sodium (intraperitoneal injection $50 \mathrm{mg} / \mathrm{kg}$ ). Subsequently, these rats underwent median sternotomy. The whole lungs (including both lobes) was dissected, separated from other tissues, washed free of blood with ice-cold saline, and placed on a sterile plastic Petri dish. Afterward, the left lung lobes of the animal lungs were preserved in $10 \%$ buffered formaldehyde solution for histopathological assessment. The right upper lung tissues were preserved for W/Dc ratio assay, and the right lower lung tissues were frozen in liquid nitrogen for HYP assay.

\section{W/Dc ratio assay}

When blood and other contaminants were removed, the right upper lung tissue was weighed, dried in a drying oven at $60^{\circ} \mathrm{C}$ for 72 hours, and weighed again according to the method of Oliveira [18]; the W/Dc ratio is expressed as the ratio of wet lung weight (mg) to dry lung weight (mg).

\section{HYP assay}

Lung collagen deposition was estimated by measuring the HYP content of lung homogenates in accordance 


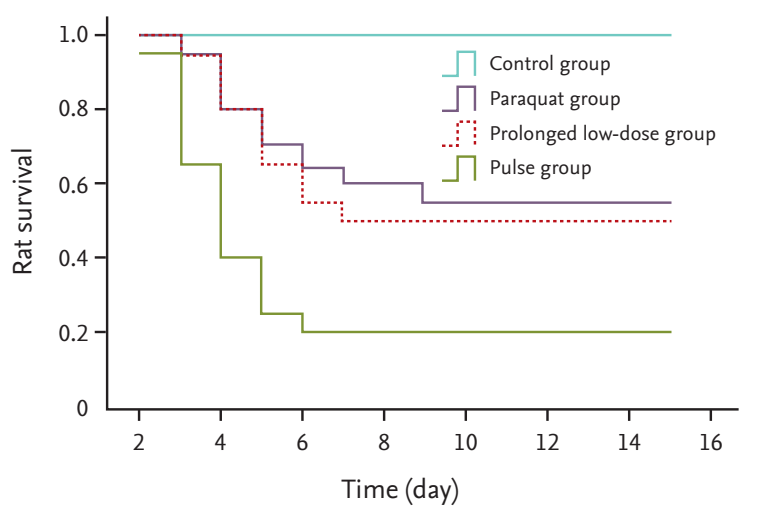

Figure 1. Survival rate analysis. Survival rates were estimated by the Kaplan-Meier method and compared by a log-rank test. Survival analysis results demonstrated a similar survival rate for prolonged low-dose group compared with the pulse group $(p>0.05)$.

with a previously described method [19]. Results are expressed in $\mu \mathrm{g}$ HYP per mg wet lung.

\section{Histological assay}

The left lung lobe samples were fixed in 10\% formalin for 24 hours, embedded in paraffin, and sectioned (thickness, $4 \mu \mathrm{m}$ ). Slides were stained with hematoxylin and eosin. Slides were examined under light microscope (CX31, Olympus, Tokyo, Japan). The entire lung section was observed at a magnification of $\times 100$. The severity of fibrotic changes was assessed by using a semiquantitative scoring system from zero (normal lung) to eight (total fibrosis) [2o]. Five randomly selected microscopic observation fields of each lung histological section were evaluated and scored independently by two investigators in blinded manner.

\section{Data analysis}

All of the data were statistically analyzed using SPSS version 19.0 (IBM Co., Armonk, NY, USA). The statistical significance of the difference between four groups of individual data (lung W/Dc ratio, HYP content, and histological score) was analyzed by one-way analysis of variance with Bonferroni post-tests for comparison between groups. Survival rates were analyzed using Kaplan-Meier method and compared using log-rank test. Lung W/Dc ratio, HYP content, and histological score are expressed as mean \pm standard deviation. Statistical

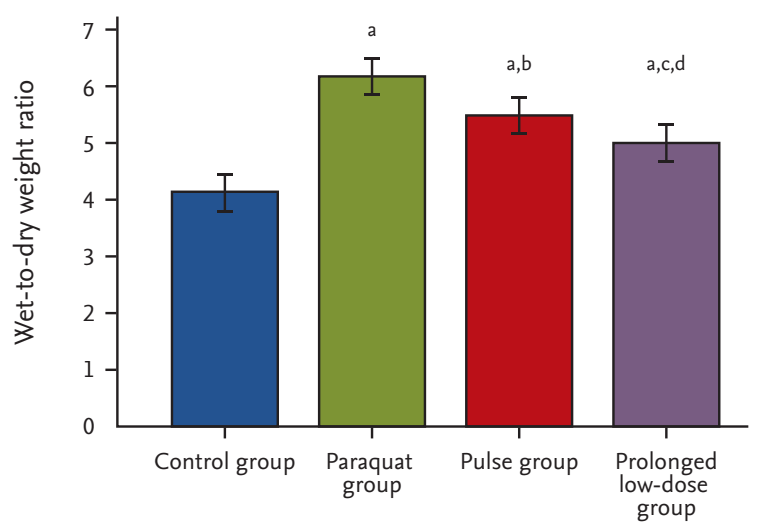

Figure 2. Effect of cyclophosphamide on wet-to-dry ratio. These ratios were lower in the prolonged low-dose group compared with that in the pulse group $(p<0.01) .{ }^{a} p<0.001$ compared with the control group, ${ }^{b} p<0.01$ and ${ }^{c} p<0.001$ compared with the paraquat group, ${ }^{\mathrm{d}} p<0.01$ compared with the pulse group.

significance was defined as $p<0.05$.

\section{RESULTS}

\section{Survival rate of rats}

At 15 days post-PQ exposure, the survival rates were $100 \%, 20 \%, 50 \%$, and $55 \%$ in the control, PQ, pulse, and prolonged low-dose groups (Fig. 1). Survival rates were higher in the prolonged low-dose group $(p<0.05)$ and pulse group $(p<0.05)$ compared with that in the PQ group. The survival rates in the prolonged low-dose group were similar to those in the pulse group $(p>0.05)$.

\section{W/Dc ratio}

At 15 days post-PQ exposure, the W/D ratios were $4.12 \pm$ $0.37,6.16 \pm 0.37,5.47 \pm 0.28$, and $4.94 \pm 0.38$ in the control, $\mathrm{PQ}$, pulse, and prolonged low-dose groups, respectively (Fig. 2). The W/D ratios in the prolonged low-dose group $(p<0.001)$ and pulse group $(p<0.01)$ were lower than that in the PQ group. These ratios were lower in the prolonged low-dose group compared with that in the pulse group $(p<0.01)$.

\section{HYP content}

At 15 days post-PQ exposure, the HYP content in the prolonged low-dose group was $3.34 \pm 0.29 \mu \mathrm{g} / \mathrm{mg}$, which was significantly lower than those in the pulse $3.65 \pm$ 


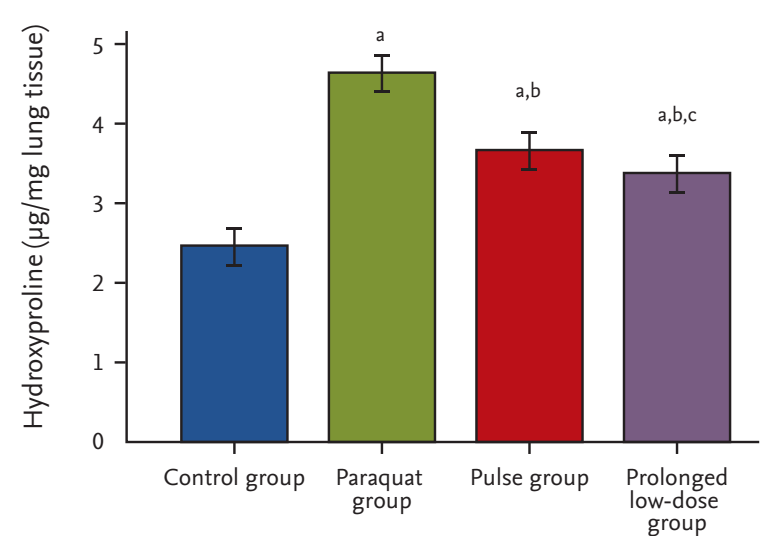

Figure 3. Effect of cyclophosphamide on hydroxyproline. The content in the prolonged low-dose group was lower than those in the pulse group $(p<0.001)$. ${ }^{\mathrm{a}} p<0.001 \mathrm{com}-$ pared with the control group, ${ }^{b} p<0.001$ compared with the paraquat group, ${ }^{c} p<0.001$ compared with the pulse group.

$0.19 \mu \mathrm{g} / \mathrm{mg}, p<0.001)$ and PQ groups $(4.67 \pm 0.12 \mu \mathrm{g} / \mathrm{mg}$, $p<0.001$ ) (Fig. 3).

\section{Histological studies}

At 15 days post-PQ exposure, the lung fibrosis score in the prolonged low-dose group was $2.69 \pm 0.84$, which was significantly lower than those in the pulse $(3.13 \pm 0.63, p<$ 0.05 ) and PQ groups (4.29 $\pm 0.47, p<0.001)$ (Figs. 4 and 5).

\section{DISCUSSION}

Lung injury is one of the most common and severe clinical sequelae of PQ poisoning, which is responsible for high mortality [3]. To date, no strong evidence is available about prolonged low-dose treatment after pulse therapy of CTX attenuating and/or reversing PQ-induced lung injury. In the present study, our result suggested that prolonged low-dose CTX after pulse therapy exerts a protective effect against PQ-induced lung injury.

In the 1980s, some studies used a CTX dose of $5 \mathrm{mg} /$ kg for PQ poisoning, and other investigations administered CTX at a dose of $15 \mathrm{mg} / \mathrm{kg}$ [21]. Given the small number of cases of $\mathrm{PQ}$ poisoning in the literature and the nonrandomized controlled trial, the use of CTX for PQ poisoning is still considered an experimental therapy. In 2013, Choi et al. [22] first approved that a CTX dose of $>15 \mathrm{mg} / \mathrm{kg}$ is effective in reducing the severity of PQ-induced lung injury, as determined by histological

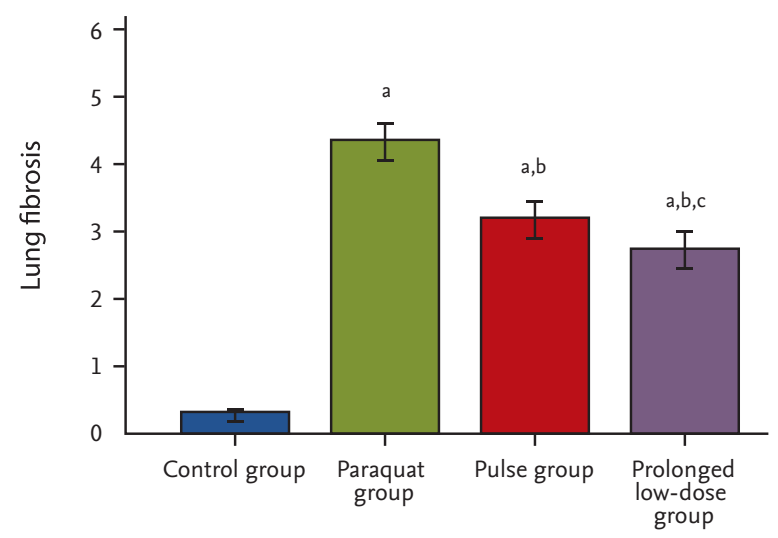

Figure 4. Effect of cyclophosphamide on lung fibrosis score. The lung fibrosis score in the prolonged low-dose group was significantly lower than those in the pulse group $(p<0.05)$. ${ }^{\mathrm{a}} \mathrm{p}<0.001$ compared with the control group, ${ }^{\mathrm{b}} \mathrm{p}<0.001 \mathrm{com}-$ pared with the paraquat group, ${ }^{c} p<0.05$ compared with the pulse group.
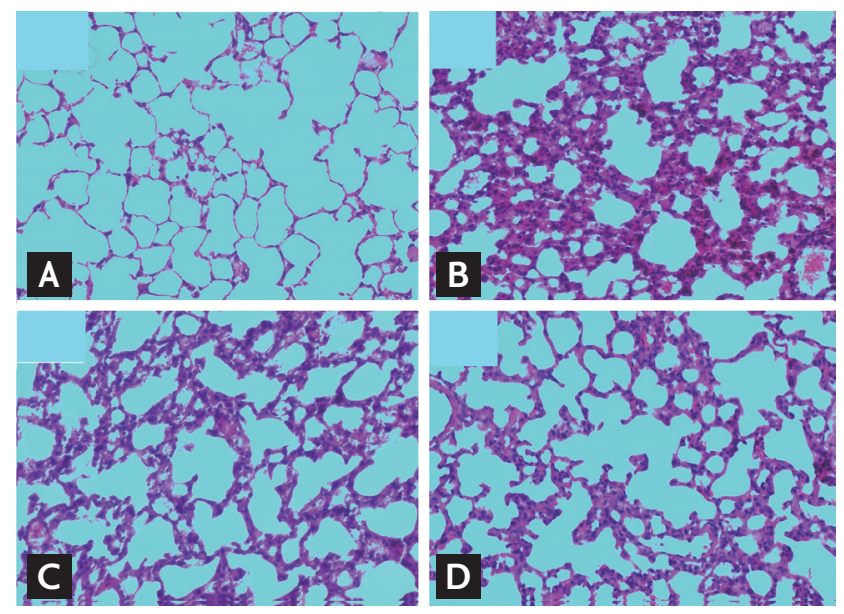

Figure 5. Effect of cyclophosphamide on pathological changes in lung tissue at 15 days post-paraquat injection ( $\times 100)$. (A) A normal lung structure and no evidence of increased alveolar wall thickness, hemorrhage, or cellular infiltration in control group. (B) Numerous inflammatory cells infiltrating the alveolar septum and spaces together with hemorrhage and congestion in paraquat group. (C) A mild decrease in inflammatory cell infiltration and alveolar wall thickness from panel in pulse group. (D) A marked decrease in inflammatory cell infiltration and alveolar wall thickness from panel in prolonged low-dose group.

and micro-computed tomography tissue examination in a rat model. These results are consistent with our histological finding. However, limited evidence exists regarding the appropriate therapeutic dose and duration of treatment.

Confronted with delayed elimination of PQ [23,24], 
which results in progressive lung damage, some studies adopted prolonged low-dose administration of CTX. Li et al. [25] demonstrated that prolonged CTX could reduce mortality and alleviate lung fibrosis. Nevertheless, this study is a retrospective research with a small sample size and short-term follow-up, which cannot completely eliminate bias. Prolonged low-dose CTX is commonly combined with glucocorticoid to attenuate lung inflammation and fibrosis in clinical. However, clinical study results are conflicting; specifically, some studies suggested a benefit for prolonged CTX combined with glucocorticoid [9-12], and other investigations failed to determine any significant difference in survival and lung fibrosis [26]. As described in a previous report [27], we hypothesize that initial CTX pulse therapies are important for rapidly suppressing PQ-induced inflammation. Moreover, prolonged low-dose CTX treatment is necessary for the progressive and sustained reduction of inflammation to reduce the toxic effect of $P Q$ and prevent lethal pulmonary inflammation. In addition, in contrast to high-dose immunosuppression, low-dose CTX treatment is inexpensive, has low toxicity, and easily administered. However, all patients should be carefully monitored during the therapeutic period.

The present study displays several limitations. First, this study failed to observe adverse effects, including dose-related leukopenia, hemorrhagic cystitis, infertility, secondary malignancies, and pulmonary toxicity [28]; these effects are commonly reversible, and they disappear when the CTX is discontinued. In addition, our research involves a small number of animals and a shortterm observation.

In conclusion, the current study is the first to suggest that prolonged CTX may attenuate the PQ-induced lung injury. However, further studies are needed to determine whether prolonged low-dose CTX could be used to protect against $\mathrm{PQ}$-induced lung toxicity in humans.

\section{KEY MESSAGE}

1. Prolonged low-dose cyclophosphamide (CTX) is an effective treatment in paraquat (PQ) intoxicated rats.

2. Prolonged low-dose CTX attenuates PQ-induced lung injury.

\section{Conflict of interest}

No potential conflict of interest relevant to this article was reported.

\section{REFERENCES}

1. Delirrad M, Majidi M, Boushehri B. Clinical features and prognosis of paraquat poisoning: a review of 41 cases. Int J Clin Exp Med 2015;8:8122-8128.

2. Bismuth C, Garnier R, Baud FJ, Muszynski J, Keyes C. Paraquat poisoning: an overview of the current status. Drug Saf 1990;5:243-251.

3. Yamashita M, Yamashita M, Ando Y. A long-term follow-up of lung function in survivors of paraquat poisoning. Hum Exp Toxicol 2000;19:99-103.

4. Vadivelan M, Chellappan A, Suryanarayana BS. The 'golden hour' in paraquat poisoning. Toxicol Int 2014;21:339-340.

5. He X, Wang L, Szklarz G, Bi Y, Ma Q. Resveratrol inhibits paraquat-induced oxidative stress and fibrogenic response by activating the nuclear factor erythroid 2-related factor 2 pathway. J Pharmacol Exp Ther 2012;342:81-90.

6. Dinis-Oliveira RJ, Sousa C, Remiao F, et al. Sodium salicylate prevents paraquat-induced apoptosis in the rat lung. Free Radic Biol Med 2007;43:48-61.

7. Fox DA, McCune WJ. Immunosuppressive drug therapy of systemic lupus erythematosus. Rheum Dis Clin North Am 1994;20:265-299.

8. Ginsburg I. Mechanisms of neutrophil-induced parenchymal cell injury. J Leukoc Biol 1998;63:518-519.

9. Zhang Q, Wu WZ, Lu YQ, et al. Successful treatment of patients with paraquat intoxication: three case reports and review of the literature. J Zhejiang Univ Sci B 2012;13:413418.

10. Botella de Maglia J, Belenguer Tarin JE. Paraquat poisoning: a study of 29 cases and evaluation of the effectiveness of the "Caribbean scheme". Med Clin (Barc) 2000;115:530533.

11. Chomchai S, Chomchai C, Kolladarungkri T. Treatment of paraquat poisoning with cyclophosphamide/dexamethasone combination: the first two years experience at Siriraj Hospital. Siriraj Hosp Gaz 2004;4:163-170.

12. Jian XD, Guo GR, Ruan YJ, et al. Clinical study on treatment of acute paraquat poisoning. Zhonghua Lao Dong Wei Sheng Zhi Ye Bing Za Zhi 2008;26:549-552.

13. Clark DG, McElligott TF, Hurst EW. The toxicity of para- 
quat. Br J Ind Med 1966;23:126-132.

14. Ahmed AA. Protective effect of montelukast on paraquatinduced lung toxicity in rats. Biosci Trends 2009;3:63-72.

15. Ghaffari AR, Noshad H, Ostadi A, Hasanzadeh N. Effect of pulse therapy with glucocorticoid and cyclophosphamide in lung fibrosis due to paraquat poisoning in rats. Saudi Med J 2011;32:249-253.

16. Lin JL, Lin-Tan DT, Chen KH, et al. Improved survival in severe paraquat poisoning with repeated pulse therapy of cyclophosphamide and steroids. Intensive Care Med 2011;37:1006-1013.

17. Baruah U, Barmon D, Hazarika M, Deka P, Kataki AC, Shrivastava S. Continuous low-dose oral chemotherapy in recurrent and persistent carcinoma of cervix following chemoradiation: a comparative study between prolonged oral cyclophosphamide and oral Etoposide. Indian J Palliat Care 2014;20:208-211.

18. Oliveira-Junior IS, Maganhin CC, Carbonel AA, Monteiro CM, Cavassani SS, Oliveira-Filho RM. Effects of pentoxifylline on TNF-alpha and lung histopathology in HCL-induced lung injury. Clinics (Sao Paulo) 2008;63:77-84.

19. Murrell GA, Tang G, Appleyard RC, del Soldato P, Wang MX. Addition of nitric oxide through nitric oxide-paracetamol enhances healing rat achilles tendon. Clin Orthop Relat Res 2008;466:1618-1624.

20. Ashcroft T, Simpson JM, Timbrell V. Simple method of estimating severity of pulmonary fibrosis on a numerical scale. J Clin Pathol 1988;41:467-470.
21. Dinis-Oliveira RJ, Duarte JA, Sanchez-Navarro A, Remiao F, Bastos ML, Carvalho F. Paraquat poisonings: mechanisms of lung toxicity, clinical features, and treatment. Crit Rev Toxicol 2008;38:13-71.

22. Choi JS, Jou SS, Oh MH, et al. The dose of cyclophosphamide for treating paraquat-induced rat lung injury. Korean J Intern Med 2013;28:420-427.

23. Beebeejaun AR, Beevers G, Rogers WN. Paraquat poisoning-prolonged excretion. Clin Toxicol 1971;4:397-407.

24. Licker M, Schweizer A, Hohn L, Morel DR, Spiliopoulos A. Single lung transplantation for adult respiratory distress syndrome after paraquat poisoning. Thorax 1998;53:620621.

25. Li X, Gong Z, Zhang H, Tan H. Effectiveness and safety of cyclophosphamide for treating patients with paraquat-induced acute lung injury. Chin Pract Clin 2013;16:3124-3125.

26. Perriens JH, Benimadho S, Kiauw IL, Wisse J, Chee H. High-dose cyclophosphamide and dexamethasone in paraquat poisoning: a prospective study. Hum Exp Toxicol 1992;11:129-134.

27. Wu WP, Lai MN, Lin CH, Li YF, Lin CY, Wu MJ. Addition of immunosuppressive treatment to hemoperfusion is associated with improved survival after paraquat poisoning: a nationwide study. PLoS One 2014;9:e87568.

28. Fraiser LH, Kanekal S, Kehrer JP. Cyclophosphamide toxicity. Characterising and avoiding the problem. Drugs 1991;42:781-795. 